\title{
Swelling of individual cellulose nanofibrils in water, role of crystallinity: an AFM study
}

\author{
Vegar Ottesen (1) - Kristin Syverud (1)
}

Received: 26 March 2020/ Accepted: 6 October 2020/Published online: 17 October 2020

(C) The Author(s) 2020

\begin{abstract}
Atomic force microscopy (AFM) can be used to quantitatively study nanomaterials in different media, e.g. vacuum, air, or submerged in a liquid. A technique was developed to study swelling of individual cellulose nanofibrils (CNFs) using AFM. As a case study, CNFs with different degrees of crystallinity (DoC) were examined for swellability going from dry to wet (submerged in de-ionized water). Swelling was found to depend on DoC, but no
\end{abstract}

significant correlation between fibril diameter and swellability was seen. Upon introduction of de-ionized water high DoC samples $(65 \pm 2 \%)$ were found to have a diameter increase of $34 \%$ on average, whereas low DoC $(44 \pm 2 \%)$ were found to have a diameter increase of $44 \%$ on average. A tested control, consisting of platinum nanowires on silisium, did not swell.

Electronic supplementary material The online version of this article (https://doi.org/10.1007/s10570-020-03517-8) contains supplementary material, which is available to authorized users.

V. Ottesen $(\bowtie) \cdot$ K. Syverud

Department of Chemical Engineering, NTNU,

Trondheim, Norway

e-mail: vegar.ottesen@ntnu.no

K. Syverud

e-mail: kristin-syverud@ rise-pfi.no

K. Syverud

RISE PFI, Trondheim, Norway 


\section{Graphic abstract}

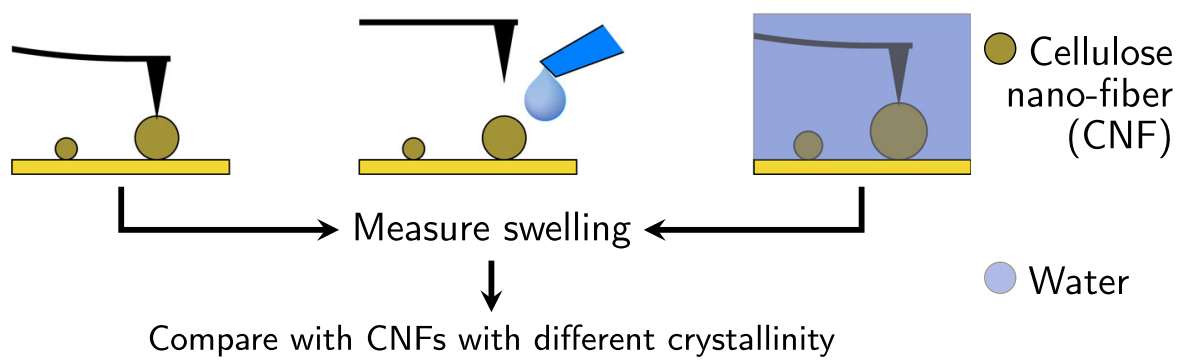

Keywords Cellulose nanofibrils $\cdot$ Swelling $\cdot$ AFM

\section{Introduction}

Swelling of wood, plant fibers and fiber networks (such as paper or textiles) is of immense importance for mechanical properties of the material in question (Benselfelt and Wagberg 2019; Mantanis et al. 1994). Swelling and deswelling of cellulose-based materials is a longstanding part of production of e.g. textiles, paper, etc.

Swelling of cotton fibers has been reported in the literature as far back as the early 1900s, where the swelling of cotton in water was reported as $27.5 \%$ (Von Hohnel 1905). Later work frequently shows similar values (Moore et al. 1950). Radial swelling of cotton fibers is typically reported as around $20 \%$ in literature today (Carr 1995). While whole fiber swelling and fiber product swelling is well discussed, little attention has been afforded the smaller constituents of the plant fiber.

As increased attention is afforded cellulose nanomaterials, the properties of these, including how such properties change in different materials, becomes an increasingly important subject both for industry and academia. Swelling of cellulose nanomaterials is, however, not at this point well discussed in the literature.

Swelling of nanocellulose and derivatives is a subject on which researchers early on provided some early theoretical work (Stone and Scallan 1968). Experimentally work has focused on films, gels and fiber networks rather than the nanofibrils themselves (Kontturi et al. 2013, 2011; Xu et al. 2016; Torstensen et al. 2018a; Benselfelt and Wagberg 2019). To our knowledge no experimental examination of the swelling of individual nanofibers has been conducted, though a brief demonstration of feasibility was shown previously (Ottesen et al. 2019). The current work explores the swelling of individual cellulose nanofibers by use of atomic force microscopy (AFM). Cotton was chosen as the raw material due to the high purity of the cellulose and the potential confounding influence of hemicellulose, which is barely present in cotton (Berthold et al. 1994).

\section{Experimental}

Preparation of cellulose nanofibrils

The preparation of cellulose nanofibrils (CNFs) is further detailed elsewhere (Ottesen et al. 2019). Briefly, two groups of CNFs were prepared from cotton linters (Celsur, Spain), one with high degree of crystallinity (DoC) and one with low DoC. DoC was modified before fibrillation. Reduction in DoC was achieved by ammonia treatment followed by boiling in water. Linters were submerged in liquid anhydrous ammonia (R717, AGA) at atmospheric pressure for 8 h. After $8 \mathrm{~h}$ had elapsed cooling was stopped and ammonia was allowed to evaporate overnight. After ammonia evaporation the treated linters were boiled in de-ionized water for $5 \mathrm{~h}$ to revert them from cellulose III allomorph back to cellulose I (Ottesen et al. 2019). After boiling they were dried in air at $100{ }^{\circ} \mathrm{C}$ overnight. Cotton linters destined to become CNFs with low degree of crystallinity were treated thrice in this manner before mechanical treatment. CNFs with high DoC were made from cotton linters which were not ammonia treated, but were boiled for $5 \mathrm{~h}$ and then dried overnight at $100{ }^{\circ} \mathrm{C}$. Fibrillation was achieved by mechanical means. Linters were beaten for 10,000 revolutions in a PFI mill at $10 \%$ solids. After beating 
the linters were passed through a Masuko brand supermasscolloider at $1 \%$ solids. After grinding the linters, now at slightly below $1 \%$ solids, were homogenized using a Rannie 15 type $12.56 \times$ homogenizer 5 times. The first pass was conducted at $600 \mathrm{bar}$ pressure drop, all subsequent passes were conducted at 1 kbar pressure drop.

\section{${ }^{13} \mathrm{C}$ NMR}

DoC was measured as described previously (Ottesen et al. 2019), where spectra are presented. Briefly, Cross-Polarization Magic Angle Spinning Carbon-13 Nuclear Magnetic Resonance (CP/MAS ${ }^{13} \mathrm{C}-\mathrm{NMR}$ ) was used. Spectra were recorded in a Bruker Avance III AQS 400 SB instrument at Innventia AB, Sweden. The instrument was operated as described in Ottesen et al. (2019) using algorithms and spectral fitting techniques as described in Larsson et al. (1997) and Wickholm et al. (1998).

\section{Preparation of control sample}

A piece of a silisium wafer was introduced to a FEI Helios NanoLab DualBeam FIB (Focused Ion Beam) where the gallium ion beam was used to produce a pattern by milling straight grooves and depositing platinum nanowires. Platinum was deposited using ion-beam induced deposition (IBID) of platinum from the precursor methyl cyclopentadienyl trimethyl platinum $\left(\left(\mathrm{CH}_{3}\right)_{3} \mathrm{Pt}\left(\mathrm{CPCH}_{3}\right)\right)$.

\section{Atomic force microscopy}

The AFM used in the current work was a Veeco Multimode V AFM using an E-scanner ( $\mathrm{s} / \mathrm{n}$ 10054EVLR) and a fluid chamber with embedded tip holder. Nanoscope 8.15 software was used to operate the AFM. Veeco's software assisted tapping mode, ScanAsyst Liquid, was used. Oscillation parameters were controlled by the software, scan rate was set as befit each session. Scan area was, with the exception of the control micrographs, set to 12 by $12 \mu \mathrm{m}$. For the control area was set to 10 by $10 \mu \mathrm{m}$. Tips used were Bruker ScanAsyst Liquid, whether the image was taken in air or liquid. These tips have a nominal tip radius of $20 \mathrm{~nm}$ and a max of $60 \mathrm{~nm}$. Samples were first imaged in air, the tip was withdrawn and de- ionized water was added before the tip was again engaged, and a new image was recorded, approximately $30 \mathrm{~min}$ after introduction of water. The water introduced had been left to rest some time (days) in a closed glass vessel by the microscope, ensuring thermal equilibrium with the surrounding environment. Areas imaged in air and submerged in water were, to the degree possible, the same. Recorded micrographs were $1024 \times 1024$ pixels large. Tipsample convolution (see Fig. 1) is avoided by measuring fibril height above the substrate as opposed to within the plane.

\section{Data analysis}

For Image Analysis Gwyddion (Neas and Klapetek 2012), FIJI (Schindelin et al. 2012) and R (RCoreTeam 2015) were used in conjunction. Micrographs were analyzed two at a time, comparing one with the other. Comparisons were conducted between sequential micrographs recorded in air or water, ascertaining uncertainty inherent in the presented technique. Later, micrographs recorded in air were compared with micrographs recorded in water.

\section{Image processing}

AFM micrographs were levelled using median of differences row alignment. Scars were corrected using Gwyddion's 'remove scars' function. Once processed in this manner the micrographs were exported as 16 bit tiff-files from Gwyddion. Images were then opened in FIJI and aligned, compensating for any drift or other distortion between the two images. To align the micrographs a Scale Invariant Feature Transform (SIFT) feature extraction and bUnwarpJ alignment were used (Lowe 2004; Arganda-Carreras et al. 2006). The SIFT feature extraction was run using the air image as the source and the water image as the target, identifying shared features. Expected transformation was set to "affine", other parameters were kept as standard. After feature extraction, alignment is performed using bUnwarpJ in FIJI. Initial deformation was set to Very Coarse, final deformation was set to Fine or Coarse, depending on which gave the best result. Divergence and Curl weight was set to 0.2 while Landmark Weight was set to 2.0. Transformation was set to "affine" or "mono", depending on which gave the best result. All other options were set 


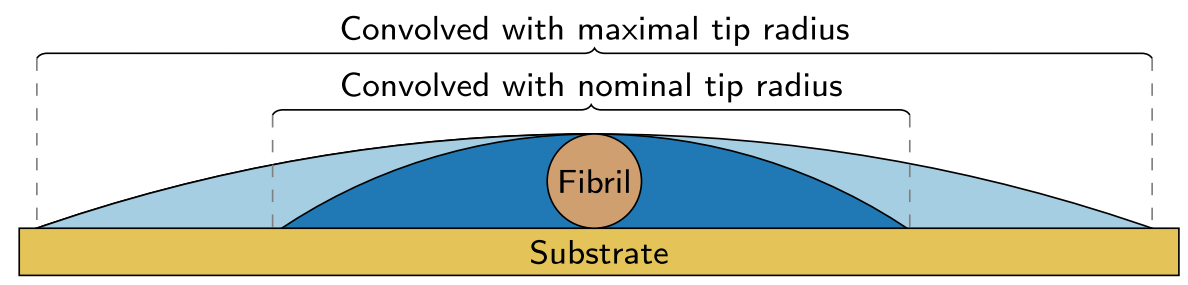

Fig. 1 AFM images within the plane show a convolution between the tip and the sample. This convolution is conceptually visualized in the top illustration, illustrating real and in-plane measured fibril diameter. The bottom illustration shows

to default values. The aligned images were cropped to exclude empty data points from further analysis. Once images were aligned cropping was conducted to eliminate empty pixels. Height profiles of fibrils were collected from the same areas on both aligned micrographs. Profiles were collected using the line selection tool with line width set to 5 pixels. Line profiles, with identifying labels, were exported as csv files from Fiji and further processed in R. Images are also saved as text images for further processing.

\section{Data processing}

Profiles are imported to $\mathrm{R}$ where they are levelled, and profile heights measured. Profiles are then compared from one image to the other, ascertaining change in fibril diameter by profile height as proxy. Differences in swelling between the tested samples was assessed using Welch two-tailed t-test, as implemented in the $\mathrm{R}$ stats library (v. 3.1.1). Swelling dependence on dry fibril diameter was assessed using a Kendall ranked correlation test—as implemented in R using the psych library (v. 1.9.12.31). Images are also imported as text images to R, where they are treated as matrices. Values of these matrices are converted from 16 bit pixel intensities to $\mathrm{nm}$ height information. One image is then subtracted from the other-the oldest micrograph (typically recorded in air) is subtracted from the newer one (typically recorded in water). Resulting positive values are interpreted as swelling and negative values seen as shrinkage. Composite images are created from these results, where positive (swelling) values are color-coded green whereas negative values (shrinkage) are color-coded red. Max intensity for either color is defined as the largest absolute value. convolved profiles assuming a fibril diameter of $3 \mathrm{~nm}$ and a rounded AFM tip with nominal and maximal tip radius of 20 and $60 \mathrm{~nm}$ respectively (reported diameters of the probes used)

\section{Results and discussion}

One technique which has the resolution to image nanofibrils with high precision in several different media is AFM. This technique has long been favored among researchers for the attainable resolution and high degree of adaptability. Unlike for example transmission electron microscopy (TEM), AFM can investigate the exact same area of a sample in different environments to see how the local sample changes when exposed to different environments. In the current study we explore how much, if at all, individual CNFs swell when submerged in de-ionized water.

It is also possible to explore dependencies of a given phenomena, such as swelling, on inherent properties of the fibril, such as degree of crystallinity (DoC).

To explore this, we recorded AFM micrographs of CNF from cotton in dry and wet state, as seen in Fig. 2. Micrographs recorded in dry and submerged state can then be compared. As AFM provides quantitative height data, we can subtract the height information from dry samples from the swollen/water laden micrographs of the same area. Any swelling would lead to positive values, while shrinking leads to negative values. Given that each micrograph is a matrix of this height information we can generate false color images which show, at a glance, whether shrinkage or swelling is occurring. Such images are shown in the image column titled "Swelling" in Fig. 2. The color coding, green for swelling and red for shrinkage, allows us to easily identify trends across the recorded data. The data reveals a clear swelling trend for both high DoC and low DoC samples, yet none for the control, Pt wires. We can also note that the fibrils appear well anchored, as fibrils which move would show up as a pair of identical fibrils—one red and one 

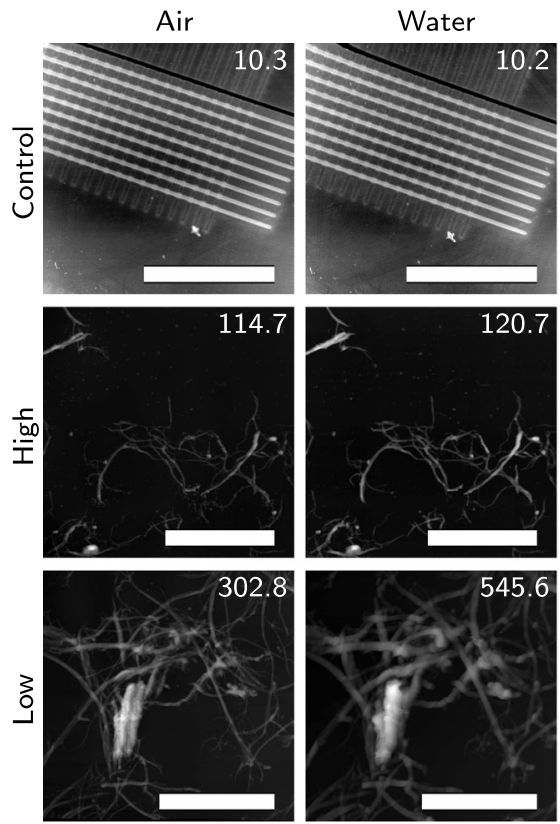

Fig. 2 Micrographs recorded in air or water shown as indicated. Column named 'Swelling' shows composite micrograph where change in height from air micrographs to water micrographs is indicated. For swelling images color indicates swelling (green) or shrinkage (red). Pixel intensity shows height or-for composite images-change in height for a given pixel. Scale

green. Any fibrils or detritus which were to leave the surface-either after introduction of water, or through interactions with the AFM tip-would show up as bright red features. Neither phenomenon is present in large amounts for any of the presented samples.

Qualitatively Fig. 2 give a clear demonstration that CNFs swell. From the images and profiles in Fig. 2 it is somewhat challenging to ascertain trends, we cannot immediately see if larger fibers swell more than smaller fibers do, or if samples with high DoC swell less than samples with low DoC. No apparent change is seen for the platinum wires, while both CNF samples show an apparent swelling. Using profiles as a basis for more quantitative analysis we can examine whether larger fibrils swell more or less than smaller ones (Fig. 3b), and if crystallinity does predict swelling of nanofibers (Fig. 3a).

Figure 3a shows an apparent dependence on DoC, which is confirmed when examined using a $t$ test $(p=$ $0.0031, t=-3.0115$ ). In terms of actual swelling the presented data shows a mean/median swelling of $34 / 30 \%$ (high DoC), and 44/45\% (low DoC). The difference in DoC between the samples is

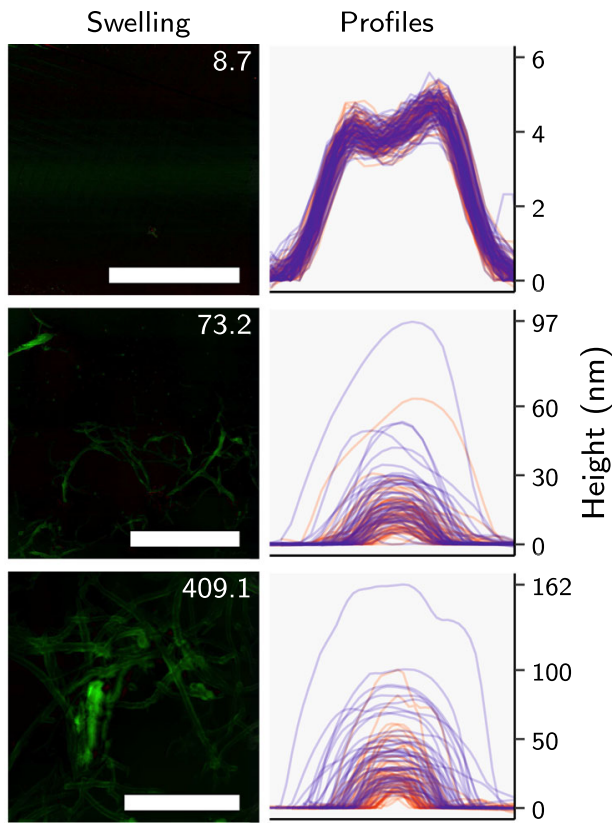

bars are $5 \mu \mathrm{m}$ long. For all images black denotes zero whereas max pixel intensity indicates a height in $\mathrm{nm}$ as shown in each image's top right corner. Column named 'profiles' shows plots shows profiles from micrographs recorded in air (red) and submerged in water (blue). There are 72 lines per color per plot

approximately $21 \%$, with the high DoC samples at $65 \pm 2 \%$ and low at $44 \pm 2 \%$. Note that a confounding factor here is that the measured crystallinity of cellulose is method-dependent (Zugenmaier 2008).

The degree of swelling shown herein can be contrasted with previously published work. In 1968 Stone and Scallan proposed theoretical values of $28 \%$ diameter change for cellulose with a $70 \%$ crystallinity, where all swelling occurs in spatially distinct amorphous cellulose shell surrounding an unswellable crystalline core. The proposed value of $28 \%$ diameter increase for a fibril is in decent agreement with other literature values for swelling of cotton fibers in water (Von Hohnel 1905; Moore et al. 1950; Carr 1995), but do not agree with the numbers presented hereinnanofibrils appear to swell more than whole fibers. One reason for this discrepancy may be that cotton fibers may have reduced capacity for radial swelling in part because of the structure of the fiber wall imposing mechanical limitations to the swellability of the fiber, limits not present for CNFs. It is also conceivable some fibrils within the whole are not water accessible in whole plant fibers, reducing swellability. Stone and 


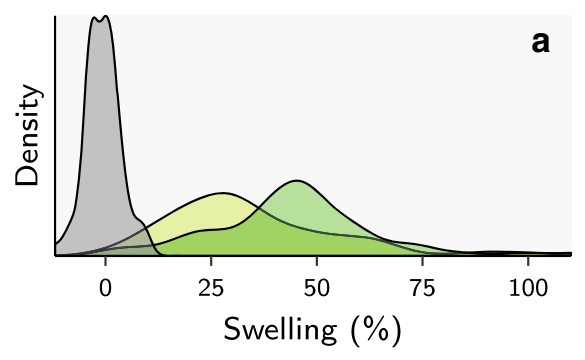

Fig. 3 Swelling of CNF by sample (a) or as a function of dry fibril diameter. a shows density plots for swelling of control samples (O), high DoC CNFs(O) and low DoC CNFs (O). Plot

Scallan, the first to discuss swelling of cellulose nanofibers, chose a structural model which permits a discussion of swelling as it pertains to DoC. If all swelling occurs in a swellable shell with a specified thickness this would, using Stone and Scallan's numbers, suggest amorphous cellulose should swell by a total of $222 \%$. The proposed value is not in agreement with experimental results; Kontturi et al. (2011) showed a height increase for an ultra-thin, amorphous cellulose film of $97.4 \%$, far below the proposed value. It is worth noting that while this discrepancy is large, the self-arranging nature of cellulose polymers suggest such thin-films are likely to have some degree of order within themselves, reducing swellability below a theoretical maximum.

Should swelling in whole fibers be constricted by the structure as a whole, a phenomenon demonstrated for films of CNF (Torstensen et al. 2018b), it is prudent to ask the same of individual CNFs. The samples examined are mechanically produced $\mathrm{CNF}$, and as such primarily consists of bundles of elementary fibrils, as opposed to individualized elementary fibrils. The reported size of elementary fibrils from cotton varies, but is commonly reported around $3.5 \mathrm{~nm}$ (Blackwell and Kolpak 1975; Zhang 1997; MartinezSanz et al. 2017). The vast majority of CNFs measured in the current work are substantially larger, viz. they are bundles of elementary fibrils. While the studied fibrils swell more than published values for whole fibers suggest, it is conceivable that this bundling may restrict swelling in the same manner hypothesized for whole fibers above. To assess whether this is the case we may examine swelling dependence on original, dry diameter; larger fibrils should swell less if this were the case. Figure 3b, where this is examined, suggests no immediately

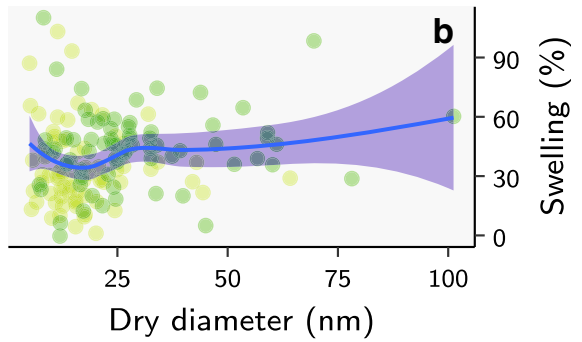

b shows dry diameter plotted against swelling in\%. Data points are shown as individual points in $\mathbf{b}$, a loess regression line is also shown with a $95 \%$ confidence interval as a ribbon

apparent correlation. Using a Kendall ranked correlation test we reach the same conclusion-no significant $(p=0.16)$ correlation is seen between dry fibril diameter and swelling in de-ionized water. Statistical considerations, with respect to both DoC and dry diameter are further expounded upon in Supplementary Materials (Table S1). This suggests any physical restriction to swelling occurs due to the structure of the whole fiber, or interactions between CNFs are not present in individual nanofibers in the studied size range.

Where the swelling occurs is of interest in terms of fundamental properties and structure of the nanofibers themselves. Our results show that swelling is inversely correlated with DoC, demonstrating that less orderered (more amorphous) cellulose will swell more than more ordered (crystalline) cellulose. From this alone we can postulate that swelling is likely to occur preferentially in volumes that are readily available to water molecules, such as surface areas, interfaces between elementary fibrils and areas with defects or increased disorder (more amorphous areas) in a given fibril. Beyond this observation the results suggest the presented method is a potential tool to address the structure of the elementary fibril; if crystalline and shorter amorphous domains are longitudinally separated along elementary fibrils, as in the historically popular fringed-fibrillar model (Hess et al. 1957; Abitz et al. 1930), swelling will also vary along a fibril's length. We would therefore expect to see periodic swelling along the fibers. We would also expect this effect to be amplified by increased water absorption in interfacial regions between crystalline and amorphous regions (Niinivaara et al. 2016). Such longitudinally distinct regions would be most prominent for elementary fibrils, whereas microfibrils 
consisting of numerous elementary fibrils may see such an effect diminish or disappear if the domains do not line up in the fibril bundle. In the case of bundles of elementary fibrils where amorphous domains are scattered, removing periodic nature of swelling we should still see some increased surface roughness of the CNF, as elementary fibrils on the surface swell more in one location than another. Such periodicity or roughness should be visible in both false color micrographs in Fig. 2. Any periodicity to the swelling should also be present as a binodal distribution of swelling in Fig. 3a. From micrographs neither swelling periodicity or increased surface roughness appears evident, and no distinct binodal distribution is selfevident in Fig. 3a. Note that the blunt tip used may obscure the hypothesized roughness, leading to this observation being of limited confidence. Further work with a substantially different tip geometry may be used to examine the question, however. While not exhaustive and conclusive, the data presented herein does not, in our view, support distinct amorphous or crystalline domains, but is consistent with the perception of cellulose fibrils having defects and dislocations along their length, rather than isolated in well-defined regions. This is consistent with reports of defect size and spacing (Nishiyama et al. 2003). While no binodal distribution is evident, a long tail is in evidence for both high and low DoC fibrils in Fig. 3a. Such a tail could be explained by variations in fibril adhesion to substrate, buckling and twisting during swelling or simply variations in the degree of order within the fibril. Besides the tail, there is a plateau evident for low DoC fibrils. One may argue that this plateau could be a concealed peak in a binodal distribution. While this is not immediately evident from false color micrographs, it is conceivable that some trend toward the fringed fibrillar model may be present for low DoC fibrils used in the current work. Verification of such is beyond the scope of the current paper, and more work would be needed to address the question thoroughly, but should it be present we envision it may have arisen from the treatments used to reduce DoC. The swelling with ammonia is likely to favor areas with point defects or somewhat higher degree of disorder than the norm in a given fibril. Such areas will be more accessible, and the effect of the ammonia should be more rapid and pronounced. As it swells and later re-arranges to the cellulose I allomorph it is conceivable that some fraction of a given fibril becomes largely or wholly amorphous. Any effect from ammonia treatment may have been amplified by the repeated treatment used to produce the low DoC linter. Should further data be acquired, and higher in-plane resolution obtained (using sharper tips) the technique employed in the current work could likely be used to shed further light on the structure, swelling and features of different classes of CNF or other, similar nanomaterials.

\section{Uncertainties}

All experimental techniques contain uncertainties, the presented work is no different. AFM is a highly precise technique, but environmental noise, noise in the instrument's electronics or some imperfection in the algorithms used in image processing can lead to uncertainties with respect to the presented data. To better account for the variations between images, regardless of surrounding medium, we compared images recorded in air with one another-and images recorded in water with one another. Plotting diameter change from one micrograph to the other (in the same medium) yields a statistical distribution seen in Fig. 4.

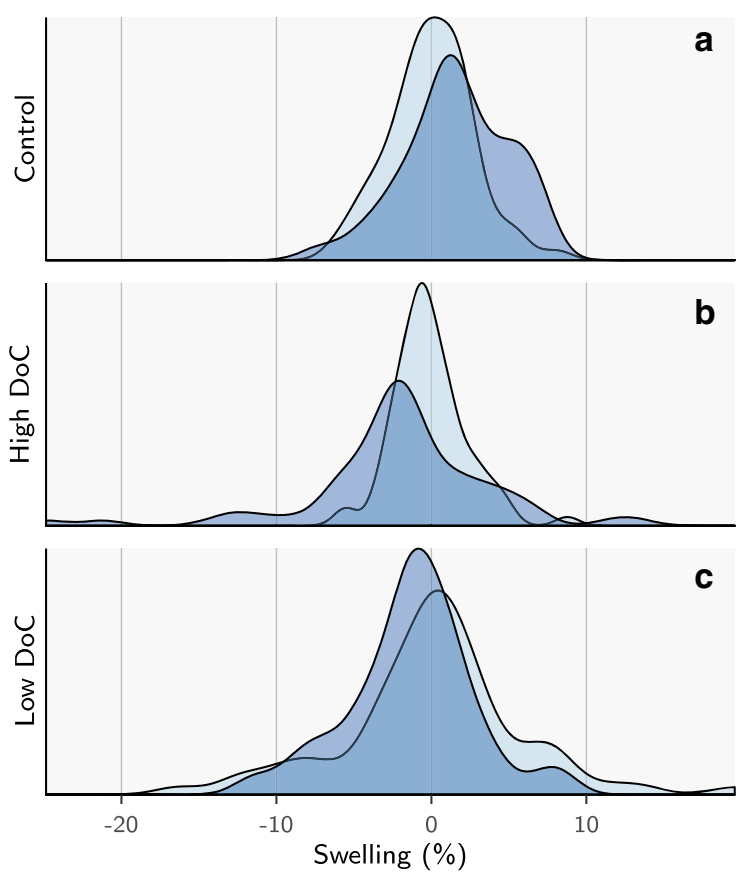

Fig. 4 Density plot of change within a given environment. Samples were checked for diameter change in sequential images recorded in air $(\bigcirc)$ or water $(O)$. Plots show results for Pt wires (a), CNF with high DoC (b) or CNF with low DoC (c) 
In the event that the cellulose-environment, cellulose-AFM tip or environment-AFM cantilever affects the measured fibril diameter we would expect the distributions from cellulose fibrils to be broader, or shifted, relative the control samples. Reasons for any observed effect here could include induced noise on the micrographs caused by addition of liquid environment, softening and subsequent deformation of CNFs while submerged, conformational changes over time in a given medium. In the event that swelling is slow, and continues after acquisition of the first micrograph, we would expect the images recorded in water to be shifted towards the right (a net increase in measured fibril diameter over time). Figure 4 shows that while the cellulose distributions are broader, they are not substantially broader than the control samples-they are also not shifted towards the right. We also note that the current setup does result in relatively broad swelling distributions around 0 , or a nearby value, for all samples in both air and water.

The distribution for CNF samples are broader than for platinum wires. The observed difference between fibrils is likely caused by the heterogeneity of the fibrils. Mechanically produced CNFs are heterogenous, and as such changes in morphology and diameter can be considered likely along any given fibril. Figure 5 considers three fibrils on a mica surface.
Figure 5a shows the middle portion of a fibril, b shows an area selected near a fibril end and c shows a portion of a fibril immediately preceding a fibril bend. Note that each profile is the average across a five pixel wide band, and each profile is shifted only one pixel relative the previous. Despite this, clear changes in profile maximum, hence reported fibril diameter, are seen from profile to profile.

Morphology, and its variation, is of importance when considering the behavior of CNF. The two qualities of CNF used herein appear indistinguishable in that regard, though DoC is seen to vary. However, as discussed in previous work (Ottesen et al. 2019) the ammonia treatment may have had unintended consequences, e.g. some small amount of cellulose II was found using ${ }^{13} \mathrm{C}$ NMR (see Ottesen et al. 2019). Moreover, results herein may indicate a possible alteration of the ultrastructure of the fibril, conceivably introducing dislocations and faults in a nonuniform manner length-wise along the treated material. Furthermore, while cotton is highly pure cellulose around one percent hemicellulose may be present (Rydholm 1965; Sczostak 2009), which could introduce some uncertainty in the swelling measurements. We consider this to be a minor concern given the purity of the material, and the variations introduced by other factors such as structural heterogeneity and
Fig. 5 Three fibrils chosen on AFM micrograph for High DoC CNFs in air. Eight adjacent profiles were drawn on each, each profile is five pixels wide and shifted one pixel along the fibril. A color gradient from black (first profile) to blue (last profile) is used to ease visualization of progression. A mark is added on left axis of each plot to denote max value (reported fibril diameter) of each curve. Scale bar is $5 \mu \mathrm{m}$ long
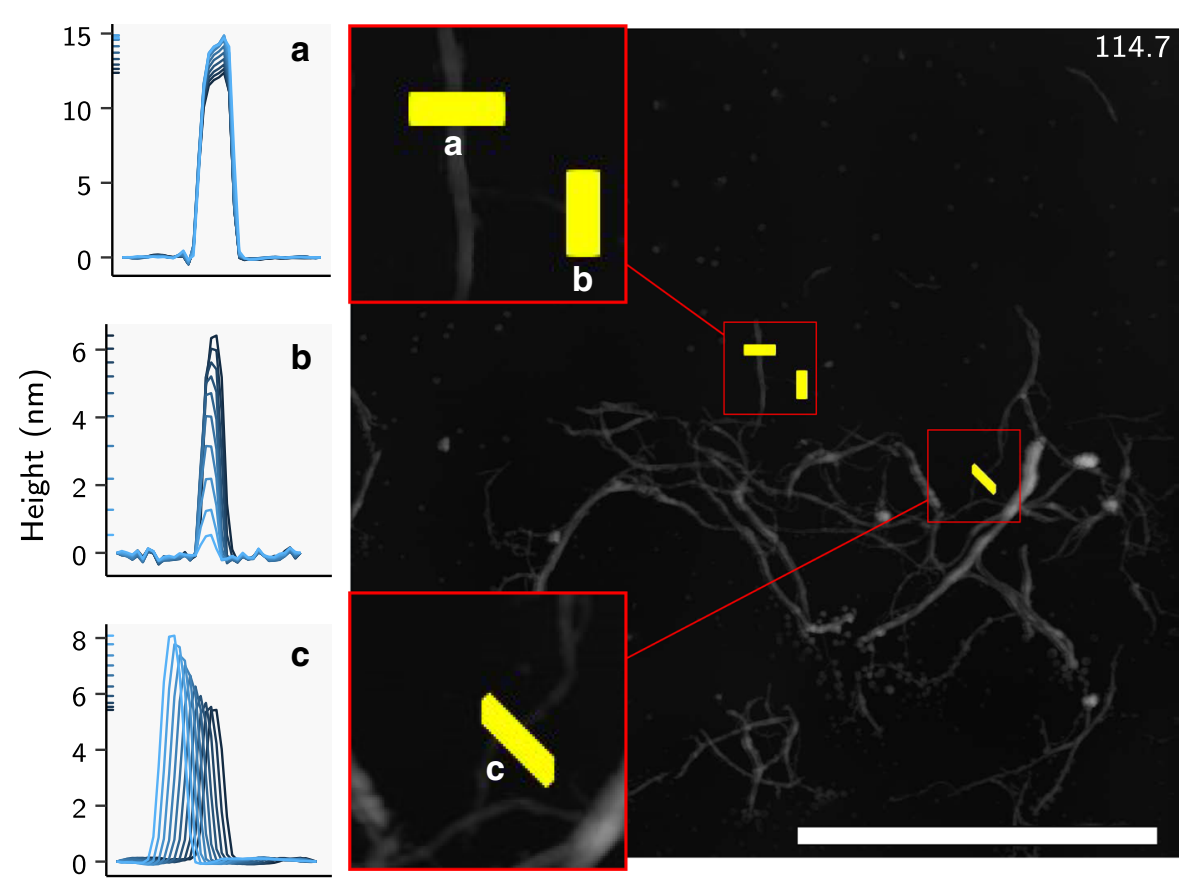
possible imperfect image overlap during analysis (as previously discussed).

These variations are important to note for experimentalists working on CNFs. The materials are structurally and morphologically heterogenous, so when fibril diameter is reported it is important to base these numbers on numerous measurements, to account for the variability inherent in the material. For experimental setups where sequential images are overlaid one another this becomes highly relevant for other, related reasons. Due to the way an AFM works it may not scan the exact same area on both passes, a slight drift is introduced by the piezoelectric scanners' hysteresis; the AFM tip may not hit the exact same place on multiple subsequent passes, much less so after introduction of a new medium. Furthermore, the alignment of the two images may be good, but if it is not pixel-perfect there is a chance it will introduce slight differences in measured height across a given fibril at a given point. As we see for the adjacent profiles in Fig. 5 relative the profiles for platinum wires in Fig. 2, the heterogeneity of CNFs can be expected to introduce uncertainties in these measurements. These results clearly underscore the value of numerous and judicious measurements when characterizing heterogenous materials like CNFs.

\section{Limitations and potential}

The first experimental step conducted herein is to dry the fibrils on mica. This drying may reduce swellability, due to the swelling hysteresis of cellulose (Morrison and Dzieciuch 1959). We show the swelling of fibrils does to be correlate with DoC in our samples, but the heterogeneity of CNF, the variation in swelling between fibrils and the noise and uncertainty present in the recorded AFM data-as previously discussedmakes the precise association difficult to determine. While AFM is suitable for studies of cellulose swelling in DI water or other liquid media or atmospheric compositions, the diameter of individual nanofibrils are frequently sufficiently small so that an uncertainty of a few A quickly represents a substantial percentage of original fibril diameter. As such AFM is indeed suitable for the experiments described, but experimentalists need to take care to measure sufficient points, and know the noise in the system, if precise measurements are to be produced. Conversely, AFM opens exciting realms for experimentalists interested in the properties of nanomaterials such as CNFs in different media. Besides spacial resolution AFM offers the ability to probe mechanical properties with high resolution, both in terms of the measured forces as well as in a spatial sense.

\section{Concluding remarks}

We have shown that swelling does occur on individual nanofibrils produced from cotton linter, and that AFM is a suitable technique to track, on the level of individual fibrils, the effect of environmental factors such as water presence. We used two classes of CNF with different degrees of crystallinity, high $(65 \pm 2)$ and low (44 \pm 2 ) —as measured using ${ }^{13} \mathrm{C}$ NMR. As the chosen raw material is nearly pure cellulose (cotton linters) contributions to swelling from other components are unlikely and are not considered in the current paper. We have found that the swelling correlates with DoC as measured using ${ }^{13} \mathrm{C}$ NMR, and that for the tested samples no correlation between (dry) nanofibril diameter and swelling was observed. Our results suggest that AFM may be used to probe the finer structure of CNF, and spatially resolve responses to environmental change, e.g. local differences in swellability along a single fibril. Our findings do not support the model of natural/un-treated cellulose nanofibrils as consisting of distinct, separated crystalline and amorphous domains.

Our main findings are, in brief;

- Swelling for CNF with a DoC of $65 \pm 2$

- Swelling for CNF with a DoC of $44 \pm 2 \%$ was found to be $44 / 45 \%$ (mean/median)

- Fibril diameter in dried state was not found to significantly correlate swellability-large and small fibers swelled to similar degrees.

- Observing swelling we found no evidence for distinct crystalline and amorphous domains in natural cellulose, though further work is necessary to properly explore this question.

- AFM is a suitable technique to investigate swelling of cellulose nanomaterials in liquid media, but care must be taken due to the structural heterogeneity of the material. 
Swelling of cotton CNFs presented in the current paper deviate from literature values for whole fibers. We suspect this may be due to physical restrictions in the fiber as a whole, or portions of potentially swellable cellulose being inaccessible to water when in a whole fiber.

Acknowledgments Cotton linter used in the experiments was provided by Celsur, Spain. Mechanical fibrillation of cotton linter was performed at RISE PFI. The Research Council of Norway is acknowledged for the support to the Norwegian Micro- and Nano-Fabrication Facility, NorFab, Project Number 245963/F50. Further funding from Dept. of chemical engineering, Norwegian University of Science and Technology (NTNU). Thanks are also extended to the community at stackexchange.com for coding advice and useful examples.

Funding Open Access funding provided by NTNU Norwegian University of Science and Technology (incl St. Olavs Hospital - Trondheim University Hospital).

Open Access This article is licensed under a Creative Commons Attribution 4.0 International License, which permits use, sharing, adaptation, distribution and reproduction in any medium or format, as long as you give appropriate credit to the original author(s) and the source, provide a link to the Creative Commons licence, and indicate if changes were made. The images or other third party material in this article are included in the article's Creative Commons licence, unless indicated otherwise in a credit line to the material. If material is not included in the article's Creative Commons licence and your intended use is not permitted by statutory regulation or exceeds the permitted use, you will need to obtain permission directly from the copyright holder. To view a copy of this licence, visit http://creativecommons.org/licenses/by/4.0/.

\section{References}

Abitz W, Gerngross O, Herrmann K (1930) Zur rontgenographischen Struk-turerforschung des Gelatinemicells. In: Naturwissenschaften. https://doi.org/10.1007/ BF01493349

Arganda-Carreras I et al (2006) Consistent and elastic registration of histological sections using vector-spline regularization, pp 85-95. ISBN 9783540462576. https://doi. org/10.1007/11889762_8

Benselfelt T, Wagberg L (2019) Unidirectional swelling of dynamic cellulose nanofibril networks: a platform for tunable hydrogels and aerogels with 3D shapeability. Biomacromolecules 20(6):2406-2412. https://doi.org/10. 1021/acs.biomac.9b00401

Berthold J et al (1994) Types of adsorbed water in relation to the ionic groups and their counter-ions for some cellulose derivatives. Polymer 35(26):5729-5736. https://doi.org/ 10.1016/S0032-3861(05)80048-5

Blackwell J, Kolpak FJ (1975) The cellulose microfibril as an imperfect array of elementary fibrils. Macromolecules 8(3):322-326. https://doi.org/10.1021/ma60045a015

Carr CM (1995) Chemistry of the textiles industry. https://doi. org/10.5860/choice.336321

Hess K, Mahl H, Gutter E (1957) Elektronenmikroskopische Darstel-lung groSSer Langsperioden in Zellulosefasern und ihr Vergleich mit den Perioden anderer Faserarten. Kolloid-Zeitschrift 155(1):1-19. https://doi.org/10.1007/ BF01501290

Kontturi E et al (2011) Amorphous characteristics of an ultrathin cellulose film. Biomacromolecules 12(3):770-777. https:// doi.org/10.1021/bm101382q

Kontturi KS, Kontturi E, Laine J (2013) Specific water uptake of thin films from nanofibrillar cellulose. J Mater Chem A 1(43):13655. https://doi.org/10.1039/c3ta12998e

Larsson PT, Wickholm K, Iversen T (1997) A CP/-MAS13C NMR investigation of molecular ordering in celluloses. Carbohydr Res 302(1-2):19-25. https://doi.org/10.1016/ S0008-6215(97)00130-4

Lowe DG (2004) Distinctive image features from scale-invariant keypoints. Int J Comput Vis 60(2):91-110. https://doi. org/10.1023/B:VISI.0000029664.99615.94

Mantanis GI, Young RA, Rowell RM (1994) Swelling of wood. Wood Sci Technol. https://doi.org/10.1007/BF00192691

Martinez-Sanz M et al (2017) Structure of cellulose microfibrils in mature cotton fibres. Carbohydr Polym 175:450-463. https://doi.org/10.1016/j.carbpol.2017.07.090

Moore AT et al (1950) The swelling of cotton in water. Text Res J 20(9):620-630. https://doi.org/10.1177/00405175500200 0904

Morrison JL, Dzieciuch MA (1959) The thermodynamic properties of the system cellulose water vapor. Can J Chem 37(9):1379-1390. https://doi.org/10.1139/v59-202

Neas D, Petr K (2012) Gwyddion: an open-source software for SPM data analysis. Open Phys. https://doi.org/10.2478/ s11534-011-0096-2

Niinivaara E et al (2016) Mimicking the humidity response of the plant cell wall by using two-dimensional systems: the critical role of amorphous and crystalline polysaccharides. Langmuir 32(8):2032-2040. https://doi.org/10.1021/acs. langmuir.5b04264

Nishiyama Y et al (2003) Periodic disorder along ramie cellulose microfibrils. Biomacromolecules 4(4):1013-1017. https://doi.org/10.1021/bm025772x

Ottesen V et al (2019) Mechanical properties of cellulose nanofibril films: effects of crystallinity and its modification by treatment with liquid anhydrous ammonia. Cellulose 26(11):6615-6627. https://doi.org/10.1007/s10570-01902546-2

R Core Team (2015) R: a language and environment for statistical computing. https://doi.org/10.1017/CBO9781107 415324.004

Rydholm SA (1965) Pulping processes, 6th edn. Interscience Publishers, New York. ISBN 978-0-471-74793-2

Schindelin J et al (2012) Fiji: an open-source platform for biological-image analysis. Nat Methods 9(7):676-682. https:// doi.org/10.1038/nmeth.2019 
Sczostak A (2009) Cotton linters: an alternative cellulosic raw material. Macromol Symposia. https://doi.org/10.1002/ masy.200950606

Stone JE, Scallan AM (1968) A structural model for the cell wall of water swollen wood fibres based on their accessibility to macromolecules. Cellul Chem Technol 2:343-358

Torstensen JO et al (2018a) Swelling and free-volume characteristics of TEMPO-oxidized cellulose nanofibril films. Biomacromolecules 19(3):1016-1025. https://doi.org/10. 1021/acs.biomac.7b01814

Torstensen JO et al (2018b) Swelling and free-volume characteristics of TEMPO-oxidized cellulose nanofibril films. Biomacromolecules 19(3):1016-1025. https://doi.org/10. 1021/acs.biomac.7b01814

Von Hohnel, FR (1905) Die Mikroskopie der Technisch Verwendeten Faser-stoffe. Ein Lehr-und Handbuch der Mikroskopischen Untersuchung der Faser-stoffe, Gewebe und Papiere. Wien \& Leipzig: Hartleben

Wickholm K, Larsson PT, Iversen T (1998) Assignment of noncrystalline forms in cellulose I by CP/MAS 13C NMR spectroscopy. Carbohydr Res 312(3):123-129. https://doi. org/10.1016/S0008-6215(98)00236-5

$\mathrm{Xu} \mathrm{Z}$ et al (2016) Morphological and swelling behavior of cellulose nanofiber (CNF)/poly(vinyl alcohol) (PVA) hydrogels: poly(ethylene glycol) (PEG) as porogen. RSC Adv 6(49):43626-43633. https://doi.org/10.1039/c6ra03620a

Zhang YZ (1997) Size and arrangement of elementary fibrils in crystalline cellulose studied with scanning tunneling microscopy. J Vac Sci Technol B Microelectron Nanometer Struct 15(4):1502. https://doi.org/10.1116/1. 589483

Zugenmaier P (2008) Crystalline cellulose and derivatives. Springer, Berlin, Heidelberg. ISBN 978-3-540-73933-3. https://doi.org/10.1007/978-3-540-73934-0

Publisher's Note Springer Nature remains neutral with regard to jurisdictional claims in published maps and institutional affiliations. 